Pesq. Vet. Bras. 35(7):659-663, julho 2015 DOI: $10.1590 / \mathrm{S} 0100-736 \mathrm{X} 2015000700010$

\title{
Avaliação dos métodos de otoscopia e exame do swab parasitológico no diagnóstico da otocariose canina: uma abordagem bayesiana $^{1}$
}

\author{
Rita C.S.M. Neves ${ }^{2 *}$, Lúcia A.F. Mateus³ ${ }^{3}$ Samara R. Lima ${ }^{4}$, Felipe A.C.S. Cruz \\ Jaqueline G. Rosa ${ }^{4}$, Rosa H.S. Ferraz ${ }^{4}$, Adriane J.Mendonça ${ }^{4}$ e Luciano A. Barros ${ }^{5}$
}

\begin{abstract}
Neves R.C.S.M., Lima S.R., Mateus L.A.F., Cruz F.A.C.S., Rosa J.G., Ferraz R.H.S., Mendonça A.J. \& Barros L.A. 2015. [Evaluation of methods of the otoscopy and parasitological swab in diagnosis of canine otocariose: a bayes analysis.] Avaliação dos métodos de otoscopia e exame do swab parasitológico no diagnóstico da otocariose canina: uma abordagem bayesiana. Pesquisa Veterinária Brasileira 35(7):659-663. Hospital Veterinário, Universidade Federal de Mato Grosso, Av. Fernando Corrêa da Costa 2367, Bairro Boa Esperança, Cuiabá, MT 78068-900, Brazil. E-mail: nevesrita@hotmail.com.br

Mites of the genus Otodectes are parasites found frequently in the ear of dogs and cats, being recognized as the main causers of external otitis in these animals. Three hundred and twenty dogs, deriving of the city of Cuiabá, Mato Grosso, had been examined through the otoscopy and of the examination of parasitological swab, with the objective to evaluate through the bayes'analysis for the estimate of the prevalence, sensitivity and especificity of these methods in the diagnosis of the infestation caused for the mite, using dates informative and not informative a priori. Each animal was considered an experimental unit. Of the total of examined dogs, $142(44.37 \%)$ had been presented positive for otocariosis. In 100 animals $(31.25 \%)$ the infestation was diagnosed by the two methods, in $31(9.69 \%)$ only for the examination of parasitological swab, in 11 (3.44\%) only for the otoscopy and in 178 $(55.62 \%)$ animal the result was negative for both the methods. The sensitivity of the otoscopy, when compared with the examination of parasitological swab, was considered lesser, however the especificity of tests they do not differ. The informative model with priori it demonstrated to be most reasonable and considerably reduced the interval of credibility of the estimates of the parameters. It can be concluded that Bayes'analysis is effective in the estimation of prevalence and characteristics of the diagnostic tests.
\end{abstract}

INDEX TERMS: Otodectescynotis, dog, ectoparasite, sensitivity, especificity, prevalence, bayes'analysis.

\footnotetext{
${ }^{1}$ Recebido em 23 de setembro de 2014.

Aceito para publicação em 22 de julho de 2015.

${ }^{2}$ Hospital Veterinário, Universidade Federal de Mato Grosso (UFMT), Av. Fernando Corrêa da Costa 2367, Bairro Boa Esperança, Cuiabá, MT 78068900, Brasil. *Autor para correspondência: nevesrita@hotmail.com.br

${ }^{3}$ Instituto de Biociências, UFMT, Av. Fernando Corrêa da Costa 2367, Bairro Boa Esperança, Cuiabá, MT 78068-900. E-mail: lmateus@ufmt.br

${ }^{4}$ Faculdade de Agronomia e Medicina Veterinária, UFMT, Avenida Fernando Corrêa da Costa 2367, Bairro Boa Esperança, Cuiabá, MT 78068900. E-mails: samara.rosolem.vet@gmail.com, felipeseabradacruz@ hotmail.com, perlesdepluie@gmail.com,rhsferraz@ufmt.br, adrianejm@ ufmt.br

${ }^{5}$ Faculdade de Veterinária, Universidade Federal Fluminense (UFF), Rua Vital Brazil Filho 64, Santa Rosa, Niterói, RJ 24230-340, Brasil. E-mail: lucianobarrosrj@gmail.com
}

RESUMO.- Ácaros do gênero Otodectes são parasitos encontrados frequentemente no ouvido de cães e gatos, sendo reconhecidos como os principais causadores de otite externa nesses animais. Trezentos e vinte cães, oriundos do município de Cuiabá, Mato Grosso, foram examinados através da otoscopia e do exame do swab parasitológico, com o objetivo de avaliar, através da análise bayesiana para a estimativa da prevalência, sensibilidade e especificidade destes métodos no diagnóstico da infestação causada pelo ácaro utilizando-se de dados a priori informativos e não informativos. Cada animal foi considerado uma unidade experimental. Do total de cães examinados, 142 (44,37\%) apresentaram-se positivos para otocariose. Em 100 animais $(31,25 \%)$ a infestação foi diagnosticada pelos dois 
métodos, em 31 (9,69\%) apenas pelo exame do swab parasitológico, em $11(3,44 \%)$ apenas pela otoscopia e em 178 $(55,62 \%)$ animais o resultado foi negativo por ambos os métodos. A sensibilidade da otoscopia, quando comparada ao exame do swab parasitológico, foi considerada menor, a especificidade dos testes, porém, não difere estatisticamente. 0 modelo, a priori informativo, demonstrou ser 0 mais plausível e reduziu consideravelmente o intervalo de credibilidade das estimativas dos parâmetros. Conclui-se que a análise bayesiana é efetiva na estimativa da prevalência e características dos testes diagnósticos.

TERMOS DE INDEXAÇÃO: Otodectes cynotis, cão, ectoparasito, sensibilidade, especificidade, prevalência, análise bayesiana.

\section{INTRODUÇÃO}

Os ácaros da espécie Otodectes cynotis são parasitos encontrados frequentemente no ouvido de cães e gatos. Eles são reconhecidos como os principais causadores de otite externa nesses animais e, desta forma, têm importância considerável na clínica médica de pequenos (Six et al. 2000, Lohse et al. 2002). Acredita-se que o ácaro 0 . cynotis esteja envolvido em, aproximadamente, cinco a $10 \%$ dos casos de otite externa canina (Logas1994), valor que pode ser superior no Brasil, visto que Larsson (1987), em estudos realizados na cidade de São Paulo, verificou que $24,3 \%$ das otopatias em cães e gatos deviam-se às otites parasitárias. No entanto, Gomes et al. (1998) encontraram prevalência de 81,4\% em animais de ambulatório.

Assim como outros integrantes da família Psoroptidae, estes ácaros caracterizam-se por não cavarem galerias na pele do hospedeiro, sendo, portanto, considerados como agentes etiológicos de sarnas não penetrantes e, devido à alimentação por escarificação da pele, são importantes causadores de otite externa (Rodriguez et al. 2003, Harvey et al. 2004).

Adicionalmente, é necessário enfatizar que bactérias e leveduras são frequentemente creditadas como fatores primários de otite (Rosychuk 1994). No entanto, na maioria dos casos, eles apenas participam como oportunistas e complicadores das alterações no conduto, mudanças essas causadas pelos reais fatores primários, como aumento da umidade e de cerúmen, além de intenso prurido, os quais determinam alterações no microclima da orelha, permitindo a multiplicação de bactérias e perpetuando as reações inflamatórias dentro do canal (Logas 1994).

Mesmo um número reduzido de ácaros pode ser suficiente para causar sinais clínicos. Tal fato, aliado à habilidade do ácaro de habitar toda a extensão do canal auditivo externo, pode dificultar o diagnóstico, uma vez que, no momento do exame otológico, o ácaro pode estar localizado profundamente no canal, tornando difícil o diagnóstico de outra forma que não seja por terapia de tentativa (Harvey et al. 2004).

0 diagnóstico da infestação pelo ácaro 0 . cynotis pode ser realizado por diferentes métodos, principalmente através de inspeção indireta, utilizando-se um otoscópio, através do qual o ácaro é observado movimentando-se nos meatos dos animais parasitados e, também, pela coleta de secreção otológica, com o auxílio de um swab, para posterior visualização do ácaro em microscópio estereoscópico ou em lâmina sob microscópio óptico. Pode-se ainda, submeter material obtido de lavado meatal à observação do ácaro em meio à secreção em microscópio estereoscópico (Souza et al. 2004).

Os testes de diagnóstico são essenciais para estudos epidemiológicos, fornecendo uma estimativa da verdadeira prevalência da doença (Berkvenset al. 2006). Assim, a estimativa precisa da prevalência é essencial tanto em saúde pública humana, quanto veterinária (Lewis \& Torgerson 2012). 0 desempenho de um teste de diagnóstico é geralmente representado por duas medidas: a sensibilidade (SE) e a especificidade (SP), cada uma descrevendo a capacidade do teste, de modo a refletir o verdadeiro e desconhecido "estado da doença" (Speybroeck et al. 2012).

A sensibilidade é definida como a probabilidade de um teste de diagnóstico ser positivo, dado que a amostra a ser testada é com certeza positiva para a doença. Da mesma forma, a especificidade é a probabilidade de um teste de diagnóstico ser negativo, dado que a amostra a ser testada é certamente negativa (Lewis \& Torgerson 2012). Infelizmente, SE e SP são raramente conhecidas exatamente e devem ser calculadas a partir de dados. Tradicionalmente nos testes de diagnóstico ainda se faz referência à sensibilidade e especificidade de teste como valores que são intrínsecos ao teste de diagnóstico, ou seja, constantes e universalmente aplicáveis (Berkvenset al. 2006). No entanto, fatores locais, como a baixa infecção ou a experiência do examinador podem influenciar a SE e a SP de um teste (Speybroeck et al. 2012).

Na verdade, pode-se argumentar que essa éa situação mais comum, uma vez que nenhum teste é considerado com $100 \%$ de precisão (Joseph et al.1995). Tendo em conta as características dos testes de diagnóstico, ou seja, que sensibilidade e especificidade podem ser variáveis e que nenhum padrão ouro está disponível no contexto específico. Combinar toda a informação disponível pode ser uma abordagem interessante (Speybroeck et al. 2011).

Quando os animais são testados usando mais do que um teste de diagnóstico, os resultados do teste podem ser dependentes (correlacionados) dentro das subpopulações infectadas e/ou não infectadas (Branscum et al. 2005). Os dois resultados de ensaio para um determinado animal são susceptíveis de ser correlacionados se ambos os ensaios medem um fenômeno biológico semelhante. Em particular, pode ser razoável assumir que os testes de diagnóstico utilizados serão dependentes quando eles compartilham uma base biológica semelhante (Branscum et al. 2005, Lewis \& Torgerson 2012). Na validação de múltiplos testes, a dependência condicional não deve ser ignorada quando os testes são de fato condicionalmente dependentes (Rahman et al. 2013). Finalmente, a abordagem "clássica" com um ou mais testes, assumindo a constância dos parâmetros e independência deles, pode subestimar verdadeira prevalência e, portanto, a gravidade da zoonose (Berkvens et al. 2006).

Ter as melhores estimativas de prevalência da doença e parâmetros dos testes é muito importante para as práticas clínicas e de saúde pública, tais como a sensibilidade, especificidade e valores preditivos positivos e negativos 
(Joseph et al. 1995). A estatística bayesiana permite combinar informações externas (ou seja, o conhecimento prévio) com os dados em mãos (Joseph et al. 1995, Enøe et al. 2000). Este tipo de análise nos permite atribuir distribuições de probabilidade para as nossas crenças anteriores e combiná-las com a probabilidade de dados para produzir uma distribuição de probabilidade posterior representando nossas crenças atualizadas (Habib et al. 2008). Assim, a prevalência e as características dos testes serão o resultado de uma combinação dos dados (resultados de teste) e a informação externa sobre estas características, que é o melhor que pode ser obtido (Berkvens et al. 2006).

Diante do exposto, este estudo apresenta uma análise bayesiana para avaliar os testes de swab e otoscopia para detecção de Otodectes cynotis em cães, dando-se destaque assim, ao potencial desse tipo de análise para a estimativa de prevalência do patógeno, sensibilidade e especificidade de ambos os testes em cães.

\section{MATERIAL E MÉTODOS}

Este estudo foi aprovado pelo Comitê de Ética em Pesquisa Animal da UFMT, protocolo no 23108.030616/09-3, estando de acordo com os Princípios Éticos na Experimentação Animal.

Trezentos e vinte cães foram examinados através da otoscopia e do exame do swab parasitológico, com o objetivo de se avaliar comparativamente estes métodos no diagnóstico da infestação. Cada animal foi considerado uma unidade experimental.

0 responsável pelo cão foi informado sobre o objetivo do estudo e, apenas após sua permissão, o cão era examinado e colhiase material para exame.

Para avaliação por otoscopia foi utilizado otoscópio com espéculos veterinário e zoom óptico (modelo: MacroView de 3,5V; marca: WelchAllyn ${ }^{\circledR}$ ), que permite uma visualização do conduto com aproximação da objetiva. Os cães foram posicionados e mantidos em estação, sendo o exame realizado, bilateralmente, sempre pelo mesmo examinador. Após cada utilização, os espéculos eram higienizados com solução de gluconato de clorexidine a $2 \%$ (Newton et al. 2006).

Paralelamente, realizou-se o exame do swab parasitológico, independente do resultado da otoscopia. Coletou-se secreção auricular do canal vertical, bilateralmente, fazendo-se a introdução do $s w a b$ no canal e posterior rotação de $360^{\circ}$ no sentido horário. Após a coleta, o swab com a amostra de secreção auricular foi mantido em solução conservante (álcool $70^{\circ} \mathrm{GL}$ ) para posterior exame em estereomicroscopia.

Os espécimes coletados foram identificados taxonomicamente segundo Serra-Freire \& Mello (2006). Uma amostra de vinte espécimes foi conservada em álcool $70^{\circ} \mathrm{GL}$ e encaminhada para depósito na coleção acarológica da Fundação Oswaldo Cruz.
Análise bayesiana. Neste estudo foi utilizado a abordagem bayesiana multinomial adaptada de Berkvens et al. (2006) para a estimativa da prevalência e características dos testes (sensibilidade e especificidade). As análises foram conduzidas no Programa R (R Core Team 2014), utilizando os pacotes prevalence (Devleesschauweret al. 2013) e JAGS via rjags (Plummer 2014).

A dependência condicional entre os dois métodos foi incluída

Quadro1. Estimativas bayesianas de probabilidade condicional dos métodos swab e otoscopia (oto) para detecção (D) de Otodectes cynotis em cães

\begin{tabular}{|c|c|c|}
\hline Parâmetros & $\begin{array}{c}\text { Probabilidades } \\
\text { condicionais }\end{array}$ & $\begin{array}{c}\text { Estimativa } \\
\text { do parâmetro }\end{array}$ \\
\hline$\theta 1$ : Prevalência (l & $\operatorname{Pr}(\mathrm{D}+)$ & $P=\theta 1$ \\
\hline$\theta 2: \mathrm{SE}_{\text {swab }}$ & $\operatorname{Pr}(s w a b+\mid \mathrm{D}+)$ & $S E_{s w a b}=\theta 2$ \\
\hline$\theta 3: \mathrm{SP}_{\text {swab }}^{\text {swad }}$ & $\operatorname{Pr}(s w a b-\mid \mathrm{D}-)$ & $S P_{\text {swab }}^{\text {swad }}=\theta 3$ \\
\hline$\theta 4: \mathrm{SE}_{\text {oto }}$ & $\operatorname{Pr}($ oto $+\mid \mathrm{D}+$, swab + ) & $S E_{o t o}=\theta 2 \theta 4+(1-\theta 2) \theta 5$ \\
\hline$\theta 5:$ & $\operatorname{Pr}($ oto $+\mid \mathrm{D}+$, swab-) & \\
\hline$\theta 6: \mathrm{SP}_{\text {oto }}$ & $\operatorname{Pr}$ (oto-|D-,swab-) & $S P_{o t o}=\theta 3 \theta 6+(1-\theta 3) \theta 7$ \\
\hline$\theta 7:$ & $\operatorname{Pr}($ oto-|D-,swab+) & \\
\hline
\end{tabular}

Quadro 2. Informaçõesa priori utilizadas para a análise bayesiana de probabilidade condicional dos métodos swab e otoscopia para detecção de Otodectes cynotis em cães Dunif=distribuição uniforme; Dbeta=distribuição beta

\begin{tabular}{|c|c|c|}
\hline Parâmetros & $\begin{array}{c}\text { Priori } \\
\text { informativa }\end{array}$ & $\begin{array}{l}\text { Priori não } \\
\text { informative }\end{array}$ \\
\hline Prevalência & $\begin{array}{l}\text { Dunif } \\
(0,05-0,80)^{\mathrm{a}}\end{array}$ & $\begin{array}{l}\text { Dunif } \\
(0-1)\end{array}$ \\
\hline $\begin{array}{l}\text { Probabilidade do swab } \\
\text { detectar o parasito e o } \\
\text { indivíduo estar infectado }\end{array}$ & $\begin{array}{l}\text { Dunif } \\
(0,95-1)^{b}\end{array}$ & $\begin{array}{l}\text { Dunif } \\
(0-1)\end{array}$ \\
\hline $\begin{array}{l}\text { Probabilidade do swab não } \\
\text { detectar o parasito e o } \\
\text { indivíduo não estar infectado }\end{array}$ & $\begin{array}{c}\text { Dunif } \\
(0,95-1)^{b}\end{array}$ & $\begin{array}{l}\text { Dunif } \\
(0-1)\end{array}$ \\
\hline $\begin{array}{l}\text { Probabilidade da otos copia detectar } \\
\text { o parasito, o indivíduo estar } \\
\text { infectado e o swab ser positivo }\end{array}$ & $\begin{array}{c}\text { Dbeta } \\
(17,3 ; 5,77)^{c}\end{array}$ & $\begin{array}{l}\text { Dunif } \\
(0-1)\end{array}$ \\
\hline $\begin{array}{l}\text { Probabilidade da otos copia detectar } \\
\text { parasito, o indivíduo estar } \\
\text { infectado e o swab ser negativo }\end{array}$ & $\begin{array}{l}\text { Dunif } \\
(0-0,1)^{\mathrm{b}}\end{array}$ & $\begin{array}{l}\text { Dunif } \\
(0-1)\end{array}$ \\
\hline $\begin{array}{l}\text { Probabilidade da otos copia não detectar } \\
\text { o parasito, o indivíduo não estar } \\
\text { infectado e o swab ser negativo }\end{array}$ & $\begin{array}{l}\text { Dunif } \\
(0,9-1)^{\mathrm{b}}\end{array}$ & $\begin{array}{l}\text { Dunif } \\
(0-1)\end{array}$ \\
\hline $\begin{array}{l}\text { Probabilidade da otos copia não detectar } \\
\text { o parasito, o indivíduo não estar }\end{array}$ & $\begin{array}{l}\text { Dunif } \\
(0-0,001)^{\mathrm{b}}\end{array}$ & $\begin{array}{l}\text { Dunif } \\
(0-1)\end{array}$ \\
\hline
\end{tabular}

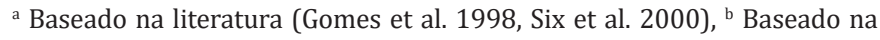
opinião de clínicos veterinários, c parâmetros alfa e beta da distribuição estimados com base na literatura considerando o valor mínimo 30\% (Larsson 1987), máximo 80\% (Frost \& Beresford-Jones 1960) e a média 65\%.

\section{Quadro 3. Resultados das estimativas dos parâmetros para os modelos com priori informativa e não informativa dos testes diagnósticos de otoscopia (oto) e swab para a detecção de Otodectes cynotis $\mathrm{SE}=$ sensibilidade; $\mathrm{SP}=$ especificidade; $\mathrm{IC}=$ intervalo de credibilidade}

\begin{tabular}{|c|c|c|c|c|c|c|c|c|}
\hline \multirow{2}{*}{$\begin{array}{l}\text { Parâmetro } \\
(\%)\end{array}$} & \multicolumn{4}{|c|}{ Priori informative } & \multicolumn{4}{|c|}{ Priori não informativa } \\
\hline & Média & Mediana & Moda & IC (95\%) & Média & Mediana & Moda & IC (95\%) \\
\hline Pre & 40,47 & 40.46 & 40,11 & 34,4 & 51,99 & 54,66 & 93,95 & $2,08-98,21$ \\
\hline $\mathrm{SE}_{\mathrm{sv}}$ & 97,46 & 97,45 & 95,32 & $95,12-99,87$ & 45,98 & 40,89 & 39,78 & $4,40-96,30$ \\
\hline $\mathrm{SP}_{\text {swab }}^{\text {swab }}$ & 97,44 & 97,40 & 95,32 & $95,12-99,87$ & 52,44 & 58,08 & 60,05 & $3,20-95,68$ \\
\hline $\mathrm{SE}_{\text {oto }}^{\text {swa }}$ & 72,83 & 72,94 & 73,24 & $65,03-79,97$ & 38,82 & 34,99 & 34,36 & $5,21-86,42$ \\
\hline $\mathrm{SP}_{\text {oto }}$ & 91,45 & 91,44 & 91,40 & $87,44-95,41$ & 60,07 & 64,43 & 65,74 & $13,04-94,77$ \\
\hline
\end{tabular}


no modelo com base em uma distribuição multinomial (Berkvens et al. 2006) e, portanto, um total de sete parâmetros tiveram de ser estimados (Quadro 1).

A informação, a priori utilizada, foi baseada na literatura e na experiência da rotina clínica veterinária (Quadro2). Para avaliar o efeito da priori nas estimativas posteriores dos parâmetros, conduzimos uma análise com uma priori não informativa e comparamos os resultados dos modelos com a priori informativa.

A verificação foi feita de acordo com os critérios descritos no Berkvens et al. (2006). Resumidamente, o Deviance Information Criterion (DIC) e número efetivo de parâmetros estimados (PD) foram usados para orientar a seleção do modelo, e o valor de $\mathrm{P}$ de Bayes foi monitorado para garantir a concordância entre a informação a priori e os dados. Assim, espera-se que quanto menor o DIC para um determinado PD, mais plausível é o modelo. Em relação ao $\mathrm{P}$ de Bayes, espera-se um valor em torno de 0.5 se há concordância entre a priori e os dados (Berkvens et al. 2006).

Os modelos foram executados com três cadeias com 50.000 interações após um corte de 5.000 iterações. Convergência de cada modelo foi avaliada por meio de gráficos de diagnóstico de convergência Gelman-Rubin e autocorrelação em rjags.

\section{RESULTADOS}

Do total de 320 cães examinados, 142 (44,37\%) apresentaram-se positivos para 0 . cynotis. Dentre os cães positivos, em $100(31,25 \%)$ animais a infestação foi diagnosticada pela otoscopia e pelo swab parasitológico, em $31(9,69 \%)$ apenas pelo exame parasitológico e em $11(3,44 \%)$ apenas pela otoscopia. Em 178 (55,62\%) animais o resultado foi negativo por ambos os métodos.

Considerando os critérios de verificação, os dois modelos convergiram (P de Bayes $\approx 0.5$ e BGR $\approx 1$ para os dois modelos). 0 número de parâmetros efetivamente estimado foi em torno de três para os dois modelos (2,83 para o modelo com priori informativa e 2,96 para o modelo com priori não informativa). Entretanto, o modelo com priori informativa foi o que obteve o menor DIC $(21,09)$ em comparação com o modelo com priori não informativa $(21,41)$. Adicionalmente, os resultados dos modelos com priori informativa e não informativa revelaram que acrescentar a informação a priori melhora as estimativas da Prevalência, SE e SP (Quadro 3). 0 modelo com priori não informativa apresentou um intervalo de credibilidade dos parâmetros consideravelmente maior do que o modelo com priori informativa.

Em relação às estimativas das características dos tes-
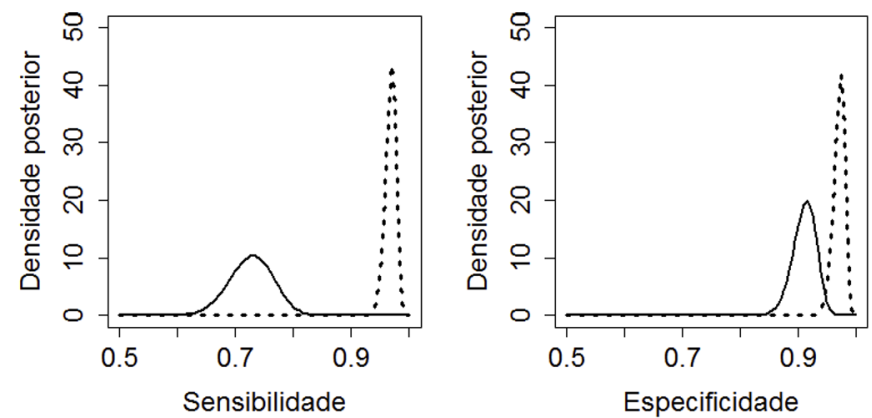

Fig.1. (A) Densidade posterior das estimativas de sensibilidade e (B) especificidade dos exames de otoscopia (linha sólida) e swab (linha pontilhada) para detecção de Otodectes cynotis em cães para o modelo com priori informativa. tes (SE e SP), a melhor combinação foi obtida para o swab, principalmente considerando a SE. A sensibilidade da otoscopia foi menor que a sensibilidade do swab, não havendo sobreposição no intervalo de credibilidade das estimativas (Fig.1A). Em relação à especificidade os dois testes são igualmente eficientes considerando a sobreposição dos seus intervalos de credibilidade (Fig.1B).

Os espécimes coletados foram conclusivos a Otodectes cynotis (Henring, 1838) (Acari: Psoroptidae) e depositados na Coleção de Artrópodes Vetores Ápteros de importância na Saúde das Comunidades (CAVAISC), do Instituto Oswaldo Cruz/FioCruz, com código de depósito entre ACA 634 e ACA 639.

\section{DISCUSSÃO}

Como no presente estudo, a otoscopia resultou em uma sensibilidade menor do que o exame do swab; destaca-se a necessidade desta ser complementada, quando negativa, em consonância com o que estabelece Larsson (1989), que considera que a otoscopia, quando positiva, seja suficiente para o diagnóstico, propiciando ganho de tempo na solução do parasitismo. Desse modo, mesmo com mais baixa sensibilidade, a otoscopia com o objetivo da detecção de Otodectes cynotis, permanece sendo útil na prática, especialmente considerando o imediatismo do diagnóstico.

Souza et al. (2004) consideram que a otoscopia também pode ser dificultada pelas condições físicas do meato acústico externo, entretanto é importante ressaltar que algumas destas situações, dentre elas o excesso de pelos nos condutos, também dificultam a passagem livre do swab, impossibilitando a retirada mecânica do ácaro, já visualizado na otoscopia.

Uma solução possível é a combinação dos resultados obtidos com os testes diferentes. De fato, a avaliação de diversas técnicas de diagnóstico e a determinação da sua sensibilidade e especificidade, com o pressuposto de que nenhuma delas pode proporcionar resultados perfeitos pode ser feito por meio da integração de várias fontes de informação (Speybroeck et al. 2011). Em análise Bayesiana na estimativa de prevalência e características do teste, uma vez que as estimativas posteriores são variáveis, dependentes tanto dos dados em mãos quanto de informações anteriores que podem ser incluídas na análise (Thomas et al. 2006).

Uma abordagem bayesiana pode ser útil neste contexto, pois permite combinar de forma flexível o conhecimento disponível a priori sobre as características dos testes de diagnóstico com novos dados (Speybroeck et al. 2012). 0 ponto importante na abordagem bayesiana é que a sensibilidade do teste e a especificidade, na verdade, também se tornam variáveis. Na abordagem clássica a sensibilidade e especificidade dos testes são parâmetros e a verdadeira prevalência é calculada a partir deles (Lesaffre et al. 2007). A circunstanciada sensibilidade e a especificidadedo teste serem variáveis, está totalmente alinhado com o espírito da análise bayesiana, onde, de fato, uma distinção entre variáveis e parâmetros deixa de existir e onde lidamos com variáveis conhecidas e desconhecidas (Lesaffre et al. 2007). Em síntese, considerar a variabilidade nas características 
dos testes diagnósticos é fundamental, pois a não contabilização de incertezas em todos os parâmetros simultaneamente, pode afetar substancialmente as inferências finais (Joseph et al. 1995), ou seja, a verdadeira prevalência.

0 conhecimento prévio pode ajudar a reduzir o número de parâmetros a serem estimados pelo modelo (Sanogo et al. 2013). 0 que pode melhorar a estimativa, como no presente estudo, onde o IC foi consideravelmente reduzido no modelo, no qual prioris informativas foram incluídas.Assim, deve ficar claro que os resultados do método bayesiano podem depender fortemente das evidências disponíveis sobre a sensibilidade e especificidade, expressa nas distribuições a priori para um SE e SP (Enøe et al. 2000, Geurden et al. 2008).

Não se deve deixar de consignar, porém, que embora útil para a análise, a informação prévia pode influenciar nas estimativas posteriores (Branscum et al. 2005, Berkvens et al. 2006). É importante, assim, ressaltar que a informação prévia incorreta pode levar a estimativas posteriores não confiáveis (Speybroeck et al. 2012). Portanto é absolutamente essencial que tal escolha possa ser justificada de forma robusta, ou seja, não é uma suposição arbitrária, pois isso pode ter uma forte influência sobre os resultados obtidos (Lewis \& Torgerson2012).

\section{CONCLUSÃO}

Conclui-se que a análise bayesiana é efetiva na estimativa da prevalência e características dos testes diagnósticos.

Agradecimentos.- Ao Dr. Gilberto Salles Gazêta, da Fundação Oswaldo Cruz, pela diagnose específica do parasito.

\section{REFERÊNCIAS}

Berkvens D., Speybroeck N., Praet N., Adel A. \& Lesaffre E. 2006. Estimating disease prevalence in a Bayesian framework using probabilistic constraints. Epidemiology 17:145-153.

Branscum A.J., Gardner I.A. \& Johnson W.O. 2005. Estimation of diagnostic-test sensitivity and specificity through Bayesian modeling. Prev. Vet. Med. 68:145-163.

Devleesschauwer B., Torgerson P., Charlier J., Levecke B., Praet N., Dorny P., Berkvens D. \& Speybroeck N. 2013. Prevalence: tools for prevalence assessment studies. R packageversion 0.2.0. Disponível em<http://cran.rproject.org/package=prevalence> Acesso em 31 jul. 2014.

Enøe C., Georgiadis M.P. \& Johnson W.O. 2000. Estimation of sensitivity and specificity of diagnostic tests and disease prevalence when the true disease state is unknown. Prev. Vet. Med. 45:61-81.

Frost R.C. \& Beresford-Jones W.P. 1960. Otodectic mange in the dog. Vet. Rec. 72:375-375.

Geurden T., Berkvens D., Casaert S., Vercruysse J. \& Claerebout E. 2008. A Bayesian evaluation of three diagnosticassays for the detection of Giardia duodenalis in symptomatic and asymptomatic dogs. Vet. Parasitol. 157:14-20.

Gomes A.P.M., Neto A.F.S., Loss Z.G., Rodriguez O.D. \& Vogel J. 1998. Sarna auricular assintomática em cães. Revta Bras. Med. Vet. 20:175-176.

Habib I., Sampers I., Uyttendaele M., De Zutter L. \& Berkvens D. 2008. A Bayesian modelling framework to estimate Campylobacter prevalence and culture methods sensitivity: application to a chicken meat survey in Belgium. J. Appl. Microbiol. 105:2002-2008.
Harvey R.G., Harari J. \& Delauche A.J. 2004. Doenças do Ouvido em cães e Gatos. Revinter, Rio de Janeiro, p.86-89.

Joseph L., Gyorkos T.W. \& Coupal L. 1995. Bayesian estimation of disease prevalence and the parameters of diagnostic tests in the absence of a gold standard. Am. J. Epidemiol. 141:263-272.

Larsson C.E. 1987. Contribuição ao Estudo das Otopatias de Cães e Gatos. Tese de Livre Docência, Faculdade de Medicina Veterinária e Zootecnia, Universidade de São Paulo, São Paulo, SP. 180p.

Larsson C.E. 1989. Dermatologia veterinária. I. Dermatites parasitárias dos carnívoros domésticos: sarnas sarcóptica, notoédricaeotoacaríase. Comun. Cient. Fac. Med. Vet. Zootec. USP 13:7-17.

Lesaffre E., Speybroeck N. \& Berkvens D. 2007. Bayes and diagnostic testing. Vet. Parasitol. 148:58-61.

Lewis F.I. \& Torgerson P.R. 2012. A tutorial in estimating the prevalence of disease in humans and animals in the absence of a gold standard diagnostic. Emerg. Themes in Epidemiol. 9:1-8.

Logas D.B. 1994. Diseases of the ear canal. Vet. Clin. North Am., Small Anim. Pract. 24:905-919.

Lohse J., Rinder H. \& Zahler M. 2002. Validity of species status of the parasitic mite otodectes cynotis. Med. Vet Entomol. 16:133-138.

Plummer M. 2014. rjags: Bayesian graphical models using MCMC. Rpackageversion 3-13. Disponível em <http://CRAN.R-project.org/ package $=$ rjags $>$ Acesso em 31 jul. 2014.

Newton H.M., Rosenkrantz W.S., Muse R. \& Griffin C.E. 2006. Evoluation of otoscope cone cleaning and desinfection produres commonly used in veterinary medical practices: a pilot study. Vet. Dermatol. 17:147-150.

Rahman A.K.M.A., Saegerman C., Berkvens D., Fretin D., Gani M.O., Ershaduzzaman M. \& Emmanuel A. 2013. Bayesian estimation of true prevalence, sensitivity and specificity of indirect ELISA, Rose Bengal Test and Slow Agglutination Test for the diagnosis of brucellosis in sheep and goats in Bangladesh. Prev. Vet. Med. 110:242-252.

RCore Team 2014. R: a language and environment for statistical computing. R Foundation for Statistical Computing, Vienna, Austria. Disponível em <http://www.R-project.org/> Acesso em 31 jul. 2014.

Rodriguez V.R.I., Ortega A., Rosado J.A. \& Bolio G.M.E. 2003. Factors affecting the prevalence of mange-mite infestations in stray dogs of Yucatán, Mexico. Vet. Parasitol. 115:61-65.

Rosychuk R. 1994. Management of otitis externa. Vet. Clin. North. Am., Small. Anim. Pract. 24:921-952.

Sanogo M., Thys E., Achi Y.L., Fretin D., Michel P., Abatih E. \& Saegerman C. 2013. Bayesian estimation of the true prevalence, sensitivity and specificity of the Rose Bengal and indirect ELISA tests in the diagnosis of bovine brucellosis. Vet. J. 195:114-120.

Serra-Freire N. \& Mello R.P. 2006. Entomologia \& Acarologia na Medicina Veterinária. L.F. Livros, Rio de Janeiro, p.41-42.

Six R.H., Clemence R.G., Thomas C.A., Behan S., Boy M.G., Watson P., Benchaoui H.A., Clements P.J., Rowan T.G. \& Jernigan A.D. 2000. Efficacy and safety of selamectin against Sarcoptesscabiei on dogs and Otodectes cynotis on dogs and cats presented as veterinary patients. Vet. Parasitol. 91:291-309.

Souza C.P., Scott F.B. \& Pereira M.J.S. 2004. Validade e reprodutibilidade da otoscopia e do reflexo otopodal no diagnóstico da infestação por Otodectes cynotis em cães. Revta Bras. Parasitol. Vet. 13:111-114.

Speybroeck N., Devleesschauwer B., Joseph L. \& Berkvens D. 2012. Misclassification errors in prevalence estimation: Bayesian handling with care. Int. J. Publ. Health 58:791-795.

Speybroeck N., Praet N., Claes F., Van Hong N., Torres K., Mao S. \& Erhart A. 2011.True versus apparent malaria infection prevalence: the contribution of a Bayesian approach. PloS One 6:1-7.

Thomas G., Dirk B., Peter G., Jozef V. \& Edwin C. 2006. A Bayesian approach for the evaluation of six diagnostic assays and the estimation of Cryptosporidium prevalence in dairy calves. Vet. Res. 37: 671-682. 